\title{
Thermophilic and Mesophilic Actinomycetes in Mouldy Hay
}

\author{
By R. CORBAZ,* P. H. GREGORY AND MAUREEN E. LACEY \\ Rothamsted Experimental Station, Harpenden, Hertfordshire
}

(Received 25 March 1963)

\begin{abstract}
SUMMARY
Actinomycetes isolated at $40^{\circ}$ and/or $60^{\circ}$ from mouldy hay included: Micromonospora vulgaris Waksman et al., Thermopolyspora polyspora Hens., $T$. glauca sp.nov., Streptomyces thermoviolaceus Hens., S. fradiae (Waksman et Curtis) Waksman et Henrici, S. griseoflavus (Krainsky) Waksman et Henrici, $\boldsymbol{S}$. olivaceus (Waksman) Waksman et Curtis, and $S$. griseus (Krainsky) Waksman et Henrici.
\end{abstract}

\section{INTRODUCTION}

Mouldy hay associated with farmer's lung disease contained large quantities of actinomycete spores; over $10^{9} / \mathrm{g}$. dry weight hay were reported by Gregory \& Lacey $(1963 a)$. This paper describes the commonest species and gives some information about their occurrence in different grades of hay.

\section{METHODS}

Actinomycetes were isolated with the aid of the Andersen sampler (Andersen, 1958) which was loaded with Petri dishes containing medium and suspended horizontally in a small wind tunnel while hay was shaken up-wind in a perforated drum (Gregory \& Lacey, 1962, 1963 $a, b)$.

Three media were used for isolation. For incubation at $40^{\circ} 0.5 \mathrm{mg}$. actidione $/ \mathrm{ml}$. was added to suppress mould growth.

Half-strength nutrient agar contained 14 g. 'Oxoid' nutrient agar granules, $10 \mathrm{~g}$. agar, 1 l. water. Yeast extract agar (Pridham et al. 1957) contained 4 g. 'Difco' yeast extract, $10 \mathrm{~g}$. malt extract, $4 \mathrm{~g}$. glucose, $20 \mathrm{~g}$. agar, $1 \mathrm{l}$. water; adjusted to pH 7.3 with KOH. V8 agar (Galindo \& Gallegly, 1960) contained $200 \mathrm{ml}$. 'V8' vegetable juice, $4 \mathrm{~g}$. calcium carbonate, $20 \mathrm{~g}$. agar, $800 \mathrm{ml}$. water; adjusted to pH $7 \cdot 3$ with $\mathrm{KOH}$.

After exposure in the Andersen sampler, Petri dishes were incubated at $40^{\circ}\left( \pm 1^{\circ}\right)$ and $60^{\circ}\left( \pm 2^{\circ}\right)$. Colonies were counted after 2, 4 and 8 days. Many isolates were studied on different media and at different temperatures. Peptone iron agar (Tresner \& Danga, 1958) containing $36 \mathrm{~g}$. peptone iron agar, $1 \mathrm{~g}$. yeast extract, 1 1. water, was used for the melanin test.

\section{RESULTS}

Although very many actinomycete colonies developed on the isolation plates, the number of different species was small: seven grew at $40^{\circ}$, of which three also grew at $60^{\circ}$. At both temperatures a few colonies without aerial mycelium developed, but

* Present address: Stations Fédérales d'Essais Agricoles, Château de Changins, Nyon, Switzerland. 
most of these showed good growth with aerial mycelium at lower temperatures. These included Streptomyces griseus (Krainsky) Waksman et Henrici; others have not yet been identified.

\section{Taxonomy}

The taxonomy of thermophilic actinomycetes is now in a state of flux, but like other workers (Tendler, 1959; Erikson, 1952; Henssen, personal communication to P.H.G., 1961) we do not regard thermophily as a good taxonomic character at the generic level. Both thermophilic and mesophilic species are included in the genus Streptomyces and we also admit thermophilic species in the genus Micromonospora. Tendler (1959) has shown that ability to grow at different temperatures is a function of nutritional requirements rather than a specific character. As morphology seems of primary importance in this group, we follow the classification of Ettlinger et al. (1958) for the identification of Streptomyces. In the following list those isolates examined in detail are indicated by ' $A$ ' and number. Typical cultures have been deposited at the Centraalbureau voor Schimmelcultures (CBS), Baarn, Netherlands, and Eidgenössische Technische Hochschule (Етн), Zürich, Switzerland.

Micromonospora vulgaris Waksman et al. Aerial mycelium abundant, cottony, white to light lavender; aerial hyphae mostly simple, emerging from the medium and often dipping back in it after a short arch over the agar (Pl. 1, fig. 4); they seem to empty when the spores are formed. Substrate mycelium light brown, sometimes darker. Spores globose, typically sharp-cornered, 0.5-0.8 $\mu$ (Pl. 1, fig. 5b), single, sessile (Pl. 1, fig. $5 a$ ). No reaction on peptone iron agar. Good and rapid growth at $40^{\circ}$ and $60^{\circ}$, no growth at $28^{\circ}$. Over 100 isolates were examined which appeared to belong to this species. Their sessile spores disagree with the description of $\boldsymbol{M}$. vulgaris, but with present knowledge of the importance and stability of the sporophore this character seems insufficient for the proposal of a new species. Isolates examined: A 64 (CBS 109.62; ЕTH 31509), A 65, etc.

Thermopolyspora polyspora Hens. Colony yellow. Aerial hyphae whitish, mostly simple, bearing lateral chains of 1 to 10 spores (Pl. 1, fig. 6). Spores globose (Pl. 1, fig. 7) $0 \cdot 8-1 \cdot 3 \mu$; also formed in medium. No reaction on peptone iron agar. No growth at $28^{\circ}$, slow vegetative growth at $40^{\circ}$, and slow vegetative and aerial growth and sporulation at $60^{\circ}$. Isolates examined: A 94 (CBS 100.63; ETH 31520), A 88, A 89, A 90, A91, А 92, A95.

Thermopolyspora glauca sp.nov. Aerial mycelium abundant, first white, becoming greenish blue and later blue grey; aerial hyphae straight, mostly simple, bearing short lateral spore chains (Pl. 1, figs. 1-3). Vegetative mycelium dark green. Spores oval, $0 \cdot 8-1 \cdot 3 \times 0 \cdot 6-0 \cdot 8 \mu$, often in pairs, sometimes single, also in chains of 3 or 4 ; some spores can be found in the medium. No reaction on peptone iron agar. Most isolates were without diffusible pigment but some produced black and some dark brown pigments. Growth very slow but good at $40^{\circ}$, and colonies were first recognizable after 5 or 6 days incubation on nutrient agar. Growth with aerial mycelium also occurs at $28^{\circ}$, but at $60^{\circ}$ there is little or no vegetative growth. Type culture: A 66 (CBS 110.62; ЕTH 28797). Other isolates examined: A 67, A 68, A 69, A70.

Henssen (1957) described a bluish thermophilic actinomycete with 4-10 spores in chains under the name of Thermoactinomyces glaucus Hens. T. monosporus (Schütze) Waksman et al. (cf. Bergey's Manual, 1948) and T. viridis Schuurmans, Olson \& 
San Clemente (1956) have both single spores and bluish aerial mycelium. Cultivated on various media our isolates never showed more than 4 spores; we therefore consider them as a new species of the genus Thermopolyspora Hens. (Micropolyspora Lechevalier, Solotorovsky \& McDurmont (1961), described four years later, is considered to be a synonym of Thermopolyspora.)

Streptomyces thermoviolaceus Hens. The description given by Henssen is completed as follows. Aerial mycelium first brown, later ash grey, not abundant, with open loops (Pl. 1, fig. 8) (cf. Henssen, 1957, figs. 7, 8). Spores oval to cylindrical, $0 \cdot 8-1 \cdot 2 \times 0 \cdot 6-0 \cdot 8 \mu$, surface covered with small hemispherical particles, $0.03 \mu(\mathrm{Pl} .1$, fig. 9), which are reported for the first time. No reaction on peptone iron agar. Growth rapid and excellent at $40^{\circ}$, good at $60^{\circ}$, and slow and usually without aerial mycelium at $28^{\circ}$. A few colonies (under $1 \%$ ) had an intense violet diffusible pigment, but as the aerial mycelium and spore morphology are identical with the other isolates they are classified under the same species. Isolates examined: A71 (CBS 111.62; ETH 28745), A 73, A74.

Streptomyces fradiae (Waksman et Curtis) Waksman et Henrici. Aerial mycelium abundant, woolly, pink. Vegetative mycelium yellowish. Spore chains in open spirals (Pl. 2, fig. 10), monopodial branching with a long straight axis. Spores smooth (Pl. 2, fig. 11), 0.7-1.0 $\times 0 \cdot 4-0 \cdot 6 \mu$. No reaction on peptone iron agar. Good growth at $40^{\circ}$, slower at $28^{\circ}$, and no growth at $60^{\circ}$. Isolates examined: A 75 (CBS 112.62; ЕTH 28746), A76.

Streptomyces griseoflavus (Krainsky) Waksman et Henrici. Aerial mycelium abundant, ash grey. Spore chains in regular spirals (P1. 2, fig. 12), monopodial branching. Spores $0 \cdot 8-1 \cdot 1 \times 0 \cdot 5-0 \cdot 7 \mu$, with short (about $0 \cdot 2 \mu$ ) spines (Pl. 2, fig. 13). No reaction on peptone iron agar. Good growth at $40^{\circ}$ and $28^{\circ}$, no growth at $60^{\circ}$. Isolates examined: A77 (CBS 113.62; ETH 31510), A78.

Streptomyces olivaceus (Waksman) Waksman et Curtis. Aerial mycelium abundant, ash grey. Spore chains in straight hyphae (Pl. 2, fig. 14), monopodial branching. Spores smooth, $0 \cdot 4-0.6 \times 0.7-1 \cdot 0 \mu(\mathrm{Pl}$. 2, fig. 15). No reaction on peptone iron agar. Good growth at $40^{\circ}$ and $28^{\circ}$, no growth at $60^{\circ}$. Isolate examined: A79 (CBS 114.62; ЕTH 28748).

Table 1 gives in diagrammatic and tabular form the diagnostic features as an aid to identification.

\section{Numbers of colonies on different media}

Tables 2 and 3 show the importance of the medium for quantitative studies of the different species of actinomycetes. Except for sample $W$ at $60^{\circ}$, many more colonies of Micromonospora vulgaris occurred on nutrient agar than on yeast agar or ' $\mathrm{V} 8$ ' agar; the opposite is true for Streptomyces thermoviolaceus and $S$. griseoflavus. More colonies of Thermopolyspora glauca developed on nutrient agar than on either yeast or ' V 8' agars. More colonies of $S$. fradiae developed on yeast agar than on nutrient agar, but 'V8' agar gave fewer colonies than nutrient agar. Although the occurrence of $T$. polyspora was not recorded in the tests on which Tables 2 and 3 are based, its abundance in self-heated mouldy hay was similar to $M$. vulgaris. Both have been isolated thousands of times and are particularly characteristic of hay associated with farmer's lung disease.

Good hay contains few actinomycetes. In mouldy hays, which have obviously heated, actinomycetes are very abundant, particularly the thermophilic species 


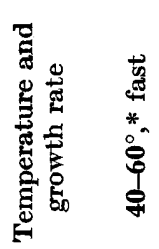
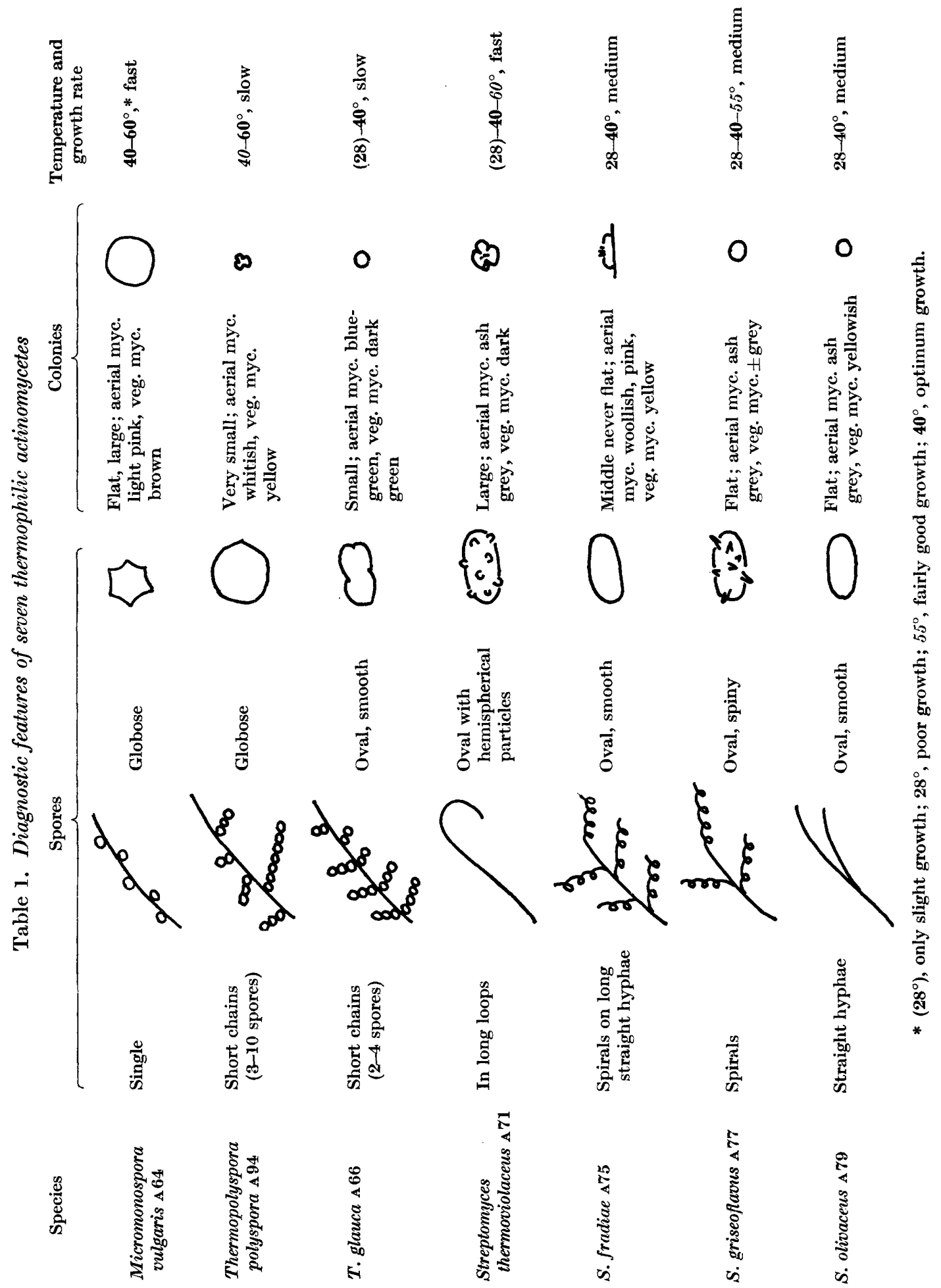
Table 2. Number of colonies on six Andersen sampler plates exposed to different hay samples shaken in the rind tunnel

\begin{tabular}{|c|c|c|c|c|c|}
\hline Hay sample & $\begin{array}{c}\text { Exposure } \\
\text { time }\end{array}$ & $\begin{array}{l}\text { Sample } \\
\text { weight }\end{array}$ & & $\begin{array}{c}\text { Micro- } \\
\text { monospora } \\
\text { vulgaris } \\
\text { No. }\end{array}$ & $\begin{array}{l}\text { Strepto- } \\
\text { myces } \\
\text { thermo- } \\
\text { violaceus } \\
\text { olonies }\end{array}$ \\
\hline 65. Farmer's lung hay & $\mathbf{5}$ & 41 & $\begin{array}{l}\mathbf{N} \\
\mathbf{Y}\end{array}$ & $\begin{array}{l}46 \\
31\end{array}$ & $\begin{array}{r}4 \\
47\end{array}$ \\
\hline 65. Farmer's lung hay & $\mathbf{5}$ & - & $\begin{array}{l}\mathbf{N} \\
\text { V } 8\end{array}$ & $\begin{array}{r}161 \\
22\end{array}$ & $\begin{array}{r}2 \\
39\end{array}$ \\
\hline Mouldy hay & 5 & 39 & $\begin{array}{l}\mathbf{N} \\
\mathbf{Y}\end{array}$ & $\begin{array}{l}236 \\
181\end{array}$ & $\begin{array}{r}15 \\
545\end{array}$ \\
\hline Mouldy hay & 5 & - & $\begin{array}{l}\text { N } \\
\text { V } 8\end{array}$ & $\begin{array}{r}192 \\
22\end{array}$ & $\begin{array}{r}1 \\
17\end{array}$ \\
\hline Mouldy hay & 5 & 52 & $\begin{array}{l}\mathbf{N} \\
\mathbf{Y}\end{array}$ & $\begin{array}{l}101 \\
134\end{array}$ & $\begin{array}{r}6 \\
218\end{array}$ \\
\hline Good hay & 15 & 41 & $\begin{array}{l}\mathbf{N} \\
\mathbf{Y}\end{array}$ & $\begin{array}{r}61 \\
4\end{array}$ & $\begin{array}{l}\mathbf{0} \\
\mathbf{6}\end{array}$ \\
\hline Good hay & 15 & 38 & $\begin{array}{l}\mathbf{N} \\
\mathbf{Y}\end{array}$ & $\begin{array}{r}22 \\
6\end{array}$ & $\begin{array}{l}2 \\
9\end{array}$ \\
\hline
\end{tabular}

$* \mathrm{~N}=$ Nutrient agar, $\mathrm{Y}=$ yeast extract agar, $\mathrm{V} 8=\mathrm{V} 8$ agar.

Table 3. Number of colonies on six Andersen sampler plates exposed to different hay samples shaken in the roind tunnel

Incubated at $40^{\circ}$. Pairs of results were from the same experiment.

\begin{tabular}{|c|c|c|c|c|c|c|c|}
\hline $\begin{array}{l}\text { Hay sample } \\
\text { reference } \\
\text { number }\end{array}$ & $\begin{array}{l}\text { Exposure } \\
\text { time } \\
\text { (sec.) }\end{array}$ & $\begin{array}{c}\text { Sample } \\
\text { weight } \\
\text { (g.) }\end{array}$ & Medium $\dagger$ & $\begin{array}{c}\text { Micro- } \\
\text { monospora } \\
\text { vulgaris }\end{array}$ & $\begin{array}{l}\text { Thermo- } \\
\text { polyspora } \\
\text { glauca }\end{array}$ & $\begin{array}{l}\text { Strepto- } \\
\text { myces } \\
\text { fradiae }\end{array}$ & $\begin{array}{c}\text { Strepto- } \\
\text { myces* } \\
\text { with } \\
\text { greyish } \\
\text { aerial } \\
\text { mycelium }\end{array}$ \\
\hline $\begin{array}{l}\text { H 65. Farmer's } \\
\text { lung hay }\end{array}$ & 5 & 41 & $\begin{array}{l}\mathbf{N} \\
\mathbf{Y}\end{array}$ & $\begin{array}{r}27 \\
3\end{array}$ & $\begin{array}{r}10 \\
5\end{array}$ & $\begin{array}{l}2 \\
4\end{array}$ & $\begin{array}{l}13 \\
61\end{array}$ \\
\hline $\begin{array}{l}\text { H 65. Farmer's } \\
\text { lung hay }\end{array}$ & 5 & - & $\begin{array}{l}\mathrm{N} \\
\text { V } 8\end{array}$ & $\begin{array}{r}30 \\
1\end{array}$ & $\begin{array}{r}20 \\
1\end{array}$ & $\begin{array}{r}21 \\
4\end{array}$ & $\begin{array}{r}2 \\
94\end{array}$ \\
\hline $\begin{array}{l}\text { H 44. Farmer's } \\
\text { lung hay }\end{array}$ & 5 & 30 & $\begin{array}{l}\mathbf{N} \\
\mathbf{Y}\end{array}$ & $\begin{array}{r}\text { c. } 700 \\
30\end{array}$ & $\begin{array}{r}69 \\
0\end{array}$ & $\begin{array}{r}4 \\
22\end{array}$ & $\begin{array}{r}89 \\
\text { c. } 900\end{array}$ \\
\hline $\begin{array}{l}\text { H 44. Farmer's } \\
\text { lung hay }\end{array}$ & 5 & - & $\begin{array}{l}\mathrm{N} \\
\mathrm{V} 8\end{array}$ & $\begin{array}{r}431 \\
0\end{array}$ & $\begin{array}{r}52 \\
1\end{array}$ & $\begin{array}{l}12 \\
26\end{array}$ & $\begin{array}{r}27 \\
\mathbf{3 3 7}\end{array}$ \\
\hline F. Mouldy hay & 5 & 39 & $\begin{array}{l}\mathbf{N} \\
\mathbf{Y}\end{array}$ & $\begin{array}{r}\text { c. } 495 \\
50\end{array}$ & $\begin{array}{r}41 \\
2\end{array}$ & $\begin{array}{r}8 \\
14\end{array}$ & $\begin{array}{r}28 \\
\text { c. } 430\end{array}$ \\
\hline F. Mouldy hay & $\mathbf{5}$ & - & $\begin{array}{l}\mathbf{N} \\
\text { V } 8\end{array}$ & $\begin{array}{r}18 \\
1\end{array}$ & $\begin{array}{l}2 \\
0\end{array}$ & $\begin{array}{r}13 \\
8\end{array}$ & $\begin{array}{r}5 \\
108\end{array}$ \\
\hline W. Mouldy hay & $\mathbf{5}$ & 52 & $\begin{array}{l}\mathbf{N} \\
\mathbf{Y}\end{array}$ & $\begin{array}{r}105 \\
39\end{array}$ & $\begin{array}{r}20 \\
2\end{array}$ & $\begin{array}{r}5 \\
21\end{array}$ & $\begin{array}{r}9 \\
\text { c. } 400\end{array}$ \\
\hline G. Good hay & 15 & 41 & $\begin{array}{l}\mathbf{N} \\
\mathbf{Y}\end{array}$ & $\begin{array}{r}12 \\
7\end{array}$ & $\begin{array}{l}0 \\
0\end{array}$ & $\begin{array}{l}\mathbf{1} \\
\mathbf{3}\end{array}$ & $\begin{array}{l}1 \\
7\end{array}$ \\
\hline SB. Good hay & 15 & 38 & $\begin{array}{l}\mathbf{N} \\
\mathbf{Y}\end{array}$ & $\begin{array}{r}19 \\
3\end{array}$ & $\begin{array}{l}\mathbf{3} \\
\mathbf{0}\end{array}$ & $\begin{array}{l}1 \\
2\end{array}$ & $\begin{array}{l}4 \\
7\end{array}$ \\
\hline
\end{tabular}

* Mainly Streptomyces thermoviolaceus and $S$. griseoflavus.

$\dagger \mathbf{N}=$ Nutrient agar, $\mathbf{Y}=$ yeast agar, V $8=$ V 8 agar. 
(Gregory \& Lacey, $1963 a$, Table 3), where grey colonies were not counted at $60^{\circ}$ because only nutrient agar was used.

We thank H.L. Nixon, R.D. Woods and A.A. Welch for the electronmicrographs.

\section{REFERENCES}

Andersen, A. A. (1958). New sampler for the collection, sizing and enumeration of viable airborne particles. J. Bact. 76, 471.

Bergey's Manual of Determinative Bacteriology (1948). 6th edition, ed. by R. S. Breed, E. G. D. Murray \& A. P. Hitchens. London and Baltimore: Ballière, Tindall \& Cox.

Erikson, D. (1952). Temperature/growth relationships of a thermophilic actinomycete, Micromonospora vulgaris. J. gen. Microbiol. 6, 286.

Etthinger, L., Corbaz, R. \& Hutter, R. (1958). Zur Systematik der Actinomyceten, 4. Eine Arteinteilung der Gattung Streptomyces Waksman et Henrici. Arch. Mikrobiol. 31, 326.

Galindo, J. \& Gallegly, M. E. (1960). The nature of sexuality in Phytophthora infestans. Phytopathology, 50, 123.

Gregory, P. H. \& LACEy, M. E. (1962). Isolation of thermophilic actinomycetes. Nature, Lond. 195, 95.

Gregory, P. H. \& LACEy, M. E. (1963a). Mycological examination of dust from mouldy hay associated with farmer's lung disease. J. gen. Microbiol. 30, 75.

Gregory, P. H. \& Lacey, M. E. (1963b). Liberation of spores from mouldy hay. Trans. Brit. mycol. Soc. 46, 1.

Henssen, A. (1957). Beiträge zur Morphologie und Systematik der thermophilen Actinomyceten. Arch. Mikrobiol. 26, 373.

Lechevalier, H. A., Solotorovsky, M. \& McDurmont, C. I. (1961). A new genus of the Actinomycetales: Micropolyspora gen.nov. J. gen. Microbiol. 26, 11.

Pridham, T. G., Anderson, P., Foley, C., Lindenfelser, L. A., Hesseltine, C. W. \& BeNEDict, R. G. (1957). A selection of media for maintenance and taxonomic study of Streptomyces. Antibiot. Ann. 1956/1957, p. 947.

Schuurmans, D. M., Olson, B. H. \& San Cremente, C. L. (1956). Production and isolation of Thermoviridin, an antibiotic produced by Thermoactinomyces viridis n.sp. Appl. Microbiol. 4, 61.

Tendler, M. D. (1959). Studies on thermophilic actinomycetes. Bull. Torrey bot. $\mathrm{Cl}$. $86,17$.

Tresner, H. D. \& Danga, F. (1958). Hydrogen sulfide production by Streptomyces as a criterion for species determination. J. Bact. 76, 239.

\section{EXPLANATION OF PLATES}

Electron micrographs of spores of Actinomycetes from mouldy hay; and photomicrographs showing aerial growth at edge of colony (photographed in air).

Plate 1

Fig. 1. Thermopolyspora glauca, edge of colony. $\times 375$.

Fig. 2. $T$. glauca, spore formation. $\times 13,500$.

Fig. 3. $T$. glauca, spores. $\times 13,500$.

Fig. 4. Micromonospora vulgaris, edge of colony. $\times \mathbf{3 7 5}$.

Fig. 5. M. vulgaris, spores. $\times 13,500$.

Fig. 6. Thermopolyspora polyspora, edge of colony. $\times 375$.

Fig. 7. T. polyspora, spores. $\times 13,500$.

Fig. 8. Streptomyces thermoviolaceus, edge of colony. $\times \mathbf{3 7 5}$.

Fig. 9. $S$. thermoviolaceus, spores. $\times 13,500$. 

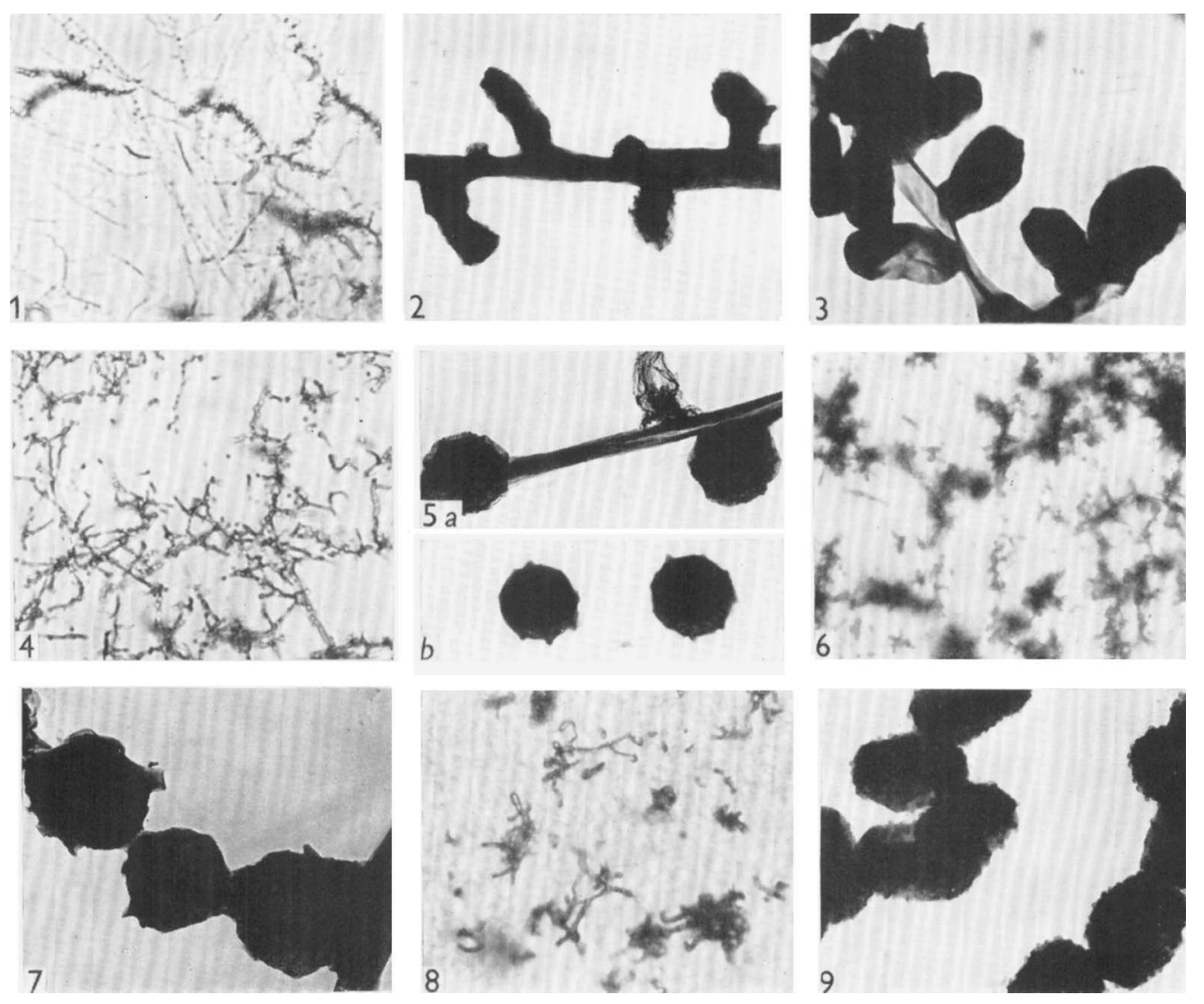

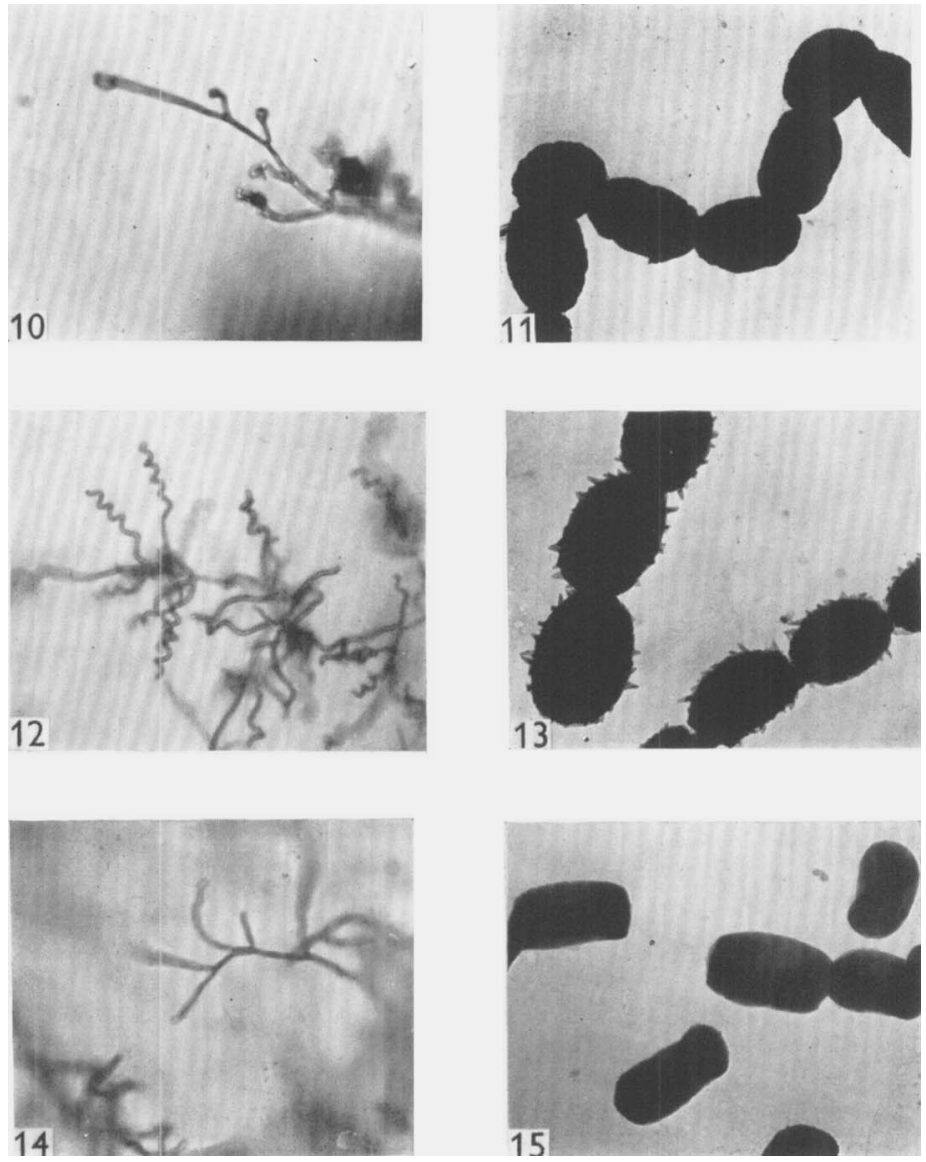

R. CORBAZ, P. H. GREGORY AND M. E. LACEY 
Plate 2

Fig. 10. S. fradiae, edge of colony. $\times \mathbf{3 7 5}$.

Fig. 11. S. fradiae, spores. $\times 13,500$.

Fig. 12. S. griseoflavus, edge of colony. $\times 375$.

Fig. 13. S. griseoflavus, spores. $\times 13,500$.

Fig. 14. S. olivaceus, edge of colony. $\times 750$.

Fig. 15. S. olivaceus, spores. $\times 13,500$. 